\title{
Estimación caudales medios y máximos de la cuenca del río Checras mediante ecuaciones sintéticas y racionales
}

\author{
Estimation of average and maximum flows of the Checras river basin through \\ synthetic and rational equations
}

Beatriz Gina Herencia Félix 1; Cesar Eduardo Carrera Saavedra ${ }^{2}$

Recibido: Junio 2019 - Aprobado: Julio 2019

\begin{abstract}
RESUMEN
La presente investigación tiene como objetivo principal integrar los parámetros morfo métricos de la cuenca con la información estadística del Servicio Nacional de Meteorología e Hidrología SENAMHI, para estimación de caudales medio y caudales máximos aplicando, respectivamente las ecuaciones sintéticas y las ecuaciones racionales.

En la primera parte se estimaron los parámetros morfo métricos dela cuenca aplicando el principio de la georreferenciación y superposición de capas vectoriales y raster, utilizando el programa Arc Gis 10.4. Luego mediante la superposición ponderada de mapas temáticos del Ministerio del Ambiente MINAM, se determina el coeficiente de escorrentía para la cuenca del rio Checras.

A continuación se procede hacer la trasformación y tratamiento estadístico de la data histórica de SENAMHI relacionado con registros de precipitación entre 1967 y 2008, obteniéndose la intensidad de precipitación de diseño y la precipitación media de la cuenca.

Con los cuatro parámetros principales identificados: área, coeficiente de escorrentía, precipitación media y la intensidad de precipitación de diseño, se procede a calcular la serie de caudales máximos así como los caudales medios y mínimos del rio Checras. Esto permite caracterizar la cuenca en términos hidrológicos y climáticos.
\end{abstract}

Palabras clave: Ecuación Sintética; parámetros morfo métricos; curvas IDF; periodos de retorno; Ecuación Racional; isohietas.

\section{ABSTRACT}

The main objective of this research is to integrate the morphometric metric parameters of the basin with the statistical information of the National Service of Meteorology and Hydrology SENAMHI, for estimating average flows and maximum flows applying, respectively, the synthetic equations and the rational equations.

In the first part the metric parameters of the basin were estimated by applying the principle of georeferencing and superposition of vector and raster layers, using the Arc Gis 10.4 program.

Then, by means of the weighted overlapping of thematic maps of the Ministry of Environment MINAM, the runoff coefficient for the Checras river basin is determined.

Then, the transformation and statistical treatment of the historical data of SENAMHI related to precipitation records between 1967 and 2008 are carried out, obtaining the intensity of design precipitation and the average precipitation of the basin.

With the four main parameters identified: area, runoff coefficient, average rainfall and design rainfall intensity, the series of maximum flows as well as the average and minimum flows of the Checras river are calculated. This allows to characterize the basin in hydrological and climatic terms.

Keywords: Synthetic Equation; metric morpho parameters; IDF curves; return periods; Rational Equation; isohietas.

\footnotetext{
${ }_{1}$ Universidad Nacional Mayor de San Marcos, Facultad de Ciencias Sociales. Lima, Perú. Geógrafa. E-mail: ginna518@gmail.com

2 Universidad Nacional Mayor de San Marcos, Facultad de Ciencias Sociales. Lima, Perú. Geógrafo. E-mail: cecarrera26@gmail.com
} 


\section{INTRODUCCIÓN}

La estimación de caudales de una cuenca es fundamental para la planificación del uso de los recursos hídricos, para la planificación agrícola y urbana así como para el dimensionamiento de las obras de infraestructura física, principalmente hidráulicas.

Se puede establecer modalidades caudales cuantificables, tales como: caudal instantáneo, caudal diario, caudal mensual, caudal promedio anual, caudal modular, caudales extremos, caudales ecológicos, caudales mínimos y máximos. A partir de estas categorías se puede generar series de caudales de diferentes tiempos de retorno así como gráficas que explican su comportamiento espaciotemporal mediante una serie de curvas tales como las curvas de duración, las curvas de variación estacional y la curva de Rippel (Villon, 2011).

En ese sentido para la estimación de caudales, se requiere información estadística histórica de precipitaciones, datos de evapotranspiración potencial y niveles de escorrentía superficial expresados en $\mathrm{m}^{3} / \mathrm{s}$ así como información paramétrica de la cuenca relacionada con su geometría y morfometría espacial.

En síntesis, para la estimación de modalidades de caudales, primero es conocer la precipitación que cae sobre la cuenca con una distribución temporal y una intensidad determinadas, luego la determinación del coeficiente de escorrentía definido por el complejo pendiente-suelovegetación así como el cálculo proyectado del área de la cuenca y otros parámetros geométricos (Robredo, 2012).

Por lo tanto, el objetivo de la presente investigación, es precisamente validar un modelo sintético y racional, para estimar los valores de flujo, principalmente de las grandes avenidas, de la cuenca del rio Checras, integrando los parámetros geométricos de la misma con los datos históricos de precipitación pluvial registrados por el SENAMHI. Entonces, la aplicación del modelo sintético y racional utilizando los datos históricos de SENAMHI permite la caracterización hidrológica y climática de la cuenca del rio Checras.

\section{METODOLOGÍA}

El proceso de investigación comprende seis (6) fases: Definición del área de estudio, georreferenciación y parametrización de la cuenca, estimación y cálculo de la escorrentía ponderada, análisis y transformación estadística de los datos históricos para estimación de precipitaciones medias y máximas, estimación del caudal medio y caudales máximos, determinación de las curvas de duración y curva masa. Finalmente la caracterización hidrológica y climática de la cuenca de Checras.

Para la presente investigación, se eligió como área de estudio, la cuenca del rio Checras que forma parte de la cuenca del rio Huaura, situado al NE de la Región LimaProvincia.

El tipo de material e información que se utilizó para hallar los parámetros morfométricos de la cuenca del rio Checras fueron imágenes satelitales Landsat 8, Carta Nacional de 1:100000, Modelo Digital de Elevación, los mismos que fueron procesados en ARCGIS aplicando el principio de Mc Harg y obteniéndose los siguientes resultados (Tabla 1$)$ :

Tabla 1. Parámetros morfométricos de la cuenca del rio Checras

\begin{tabular}{|c|c|c|c|c|}
\hline \multicolumn{3}{|c|}{ Parámetros geométricos } & \multirow{2}{*}{$\begin{array}{c}\begin{array}{c}\text { Unidad de } \\
\text { medida }\end{array} \\
\mathrm{Km} 2\end{array}$} & \multirow{2}{*}{$\begin{array}{c}\begin{array}{c}\text { Cuenca } \\
\text { Checras }\end{array} \\
820.533\end{array}$} \\
\hline \multirow{18}{*}{ 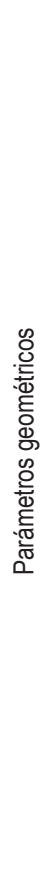 } & Área de la & & & \\
\hline & Perímetro & uenca & $\mathrm{Km}$ & 139.88 \\
\hline & Longitud $\mathrm{m}$ & & $\mathrm{Km}$ & 40.103 \\
\hline & Ancho máx & & $\mathrm{Km}$ & 31.349 \\
\hline & Ancho pron & & $\mathrm{Km}$ & 20.43 \\
\hline & Diámetro d & denca & $\mathrm{Km}$ & 32.32 \\
\hline & \multirow{4}{*}{ Centroide } & Lugar & - & $\begin{array}{c}\text { Centro poblado } \\
\text { Huancahuasi }\end{array}$ \\
\hline & & Este $\mathrm{X}$ & $\mathrm{m}$ & 311773 \\
\hline & & Norte $Y$ & $\mathrm{~m}$ & 879365 \\
\hline & & Altitud Z & $\mathrm{m}$ & 2895 \\
\hline & \multicolumn{2}{|c|}{ Factor de forma } & - & 0.51 \\
\hline & \multicolumn{2}{|c|}{ Índice de compacidad } & - & 1.37 \\
\hline & \multicolumn{2}{|c|}{ Radio de circularidad } & - & 0.527 \\
\hline & \multicolumn{2}{|c|}{ Índice de alargamiento } & - & 1.27 \\
\hline & \multicolumn{2}{|c|}{ Relación de elongación } & - & 0.086 \\
\hline & \multicolumn{2}{|c|}{ Índice asimétrico } & - & 2.219 \\
\hline & \multirow{2}{*}{$\begin{array}{l}\text { Rectángulo } \\
\text { equivalente }\end{array}$} & Lado Mayor & $\mathrm{Km}$ & 57.839 \\
\hline & & Lado menor & $\mathrm{Km}$ & 15.958 \\
\hline
\end{tabular}

A continuación se determinó el coeficiente de escorrentía de la cuenca del rio Checras, con el programa ArcGis. El coeficiente de escorrentía para la cuenca del rio Checras se ha estimado mediante la superposición ponderada de raster de cobertura vegetal, pendiente y zonas de vida (INGEMMET, 2008), obteniéndose un valor promedio de 0.48 y un valor ponderado de 0.54 , tal como se indica en la siguiente tabla (Tabla 2) y en el siguiente mapa (Figura 1).

El siguiente paso fue calcular la precipitación promedio de la cuenca del rio Checras, con los datos históricos de SENAMHI, entre 1966 y 2008, para un periodo de 42 años, aplicando el método aritmético y el método de las isoyetas, obteniéndose los siguientes resultados (Tabla 3, Tabla 4 y Figura 2)

Dentro de esta fase también se logró determinar las máximas precipitaciones para diferentes tiempos de duración así como las curvas de Intensidad, Duración y Frecuencia (IDF) para diferentes tiempos de retorno.

Se trabajó con data de 42 años de la serie 19662008 , generados en tres estaciones meteorológicas dentro y próximos a la cuenca del rio Checras. La precipitación media máxima obtenida de 24 horas es de $23.4 \mathrm{~mm}$, con una desviación estándar de 6.4, tal como se muestra en la Tabla 5 . 
Tabla 2. Valores parciales, promedio y ponderado del coeficiente de escorrentía

\begin{tabular}{|c|c|c|c|c|c|c|}
\hline Id & Unidad & Área(Km2) & Área (\%) & Coeficiente & Área*Coef & Prom_pond \\
\hline 1 & $\mathrm{~A} 1$ & 1.91 & 0.23 & 0.30 & 0.57377258 & \\
\hline 2 & A2 & 17.03 & 2.08 & 0.35 & 5.9617636 & \\
\hline 3 & A3 & 19.49 & 2.37 & 0.38 & 7.40513116 & \\
\hline 4 & A4 & 86.35 & 10.52 & 0.40 & 34.5381529 & \\
\hline 5 & A5 & 39.56 & 4.82 & 0.42 & 16.6153177 & \\
\hline 6 & A6 & 56.53 & 6.89 & 0.45 & 25.4397502 & $C=0.53984091$ \\
\hline 7 & A7 & 176.00 & 21.45 & 0.50 & 88.0013081 & \\
\hline 8 & A8 & 14.98 & 1.83 & 0.55 & 8.23869985 & \\
\hline 9 & A9 & 142.04 & 17.31 & 0.60 & 85.2264338 & \\
\hline 10 & A10 & 117.89 & 14.37 & 0.63 & 74.2678236 & \\
\hline \multirow[t]{2}{*}{11} & $\mathrm{~A} 11$ & 148.77 & 18.13 & 0.65 & 96.6983064 & \\
\hline & & SUM: 820.55 & SUM: 100 & PROM: 0.48 & SUM: 442.96646 & \\
\hline
\end{tabular}

Fuente: Elaboración propia

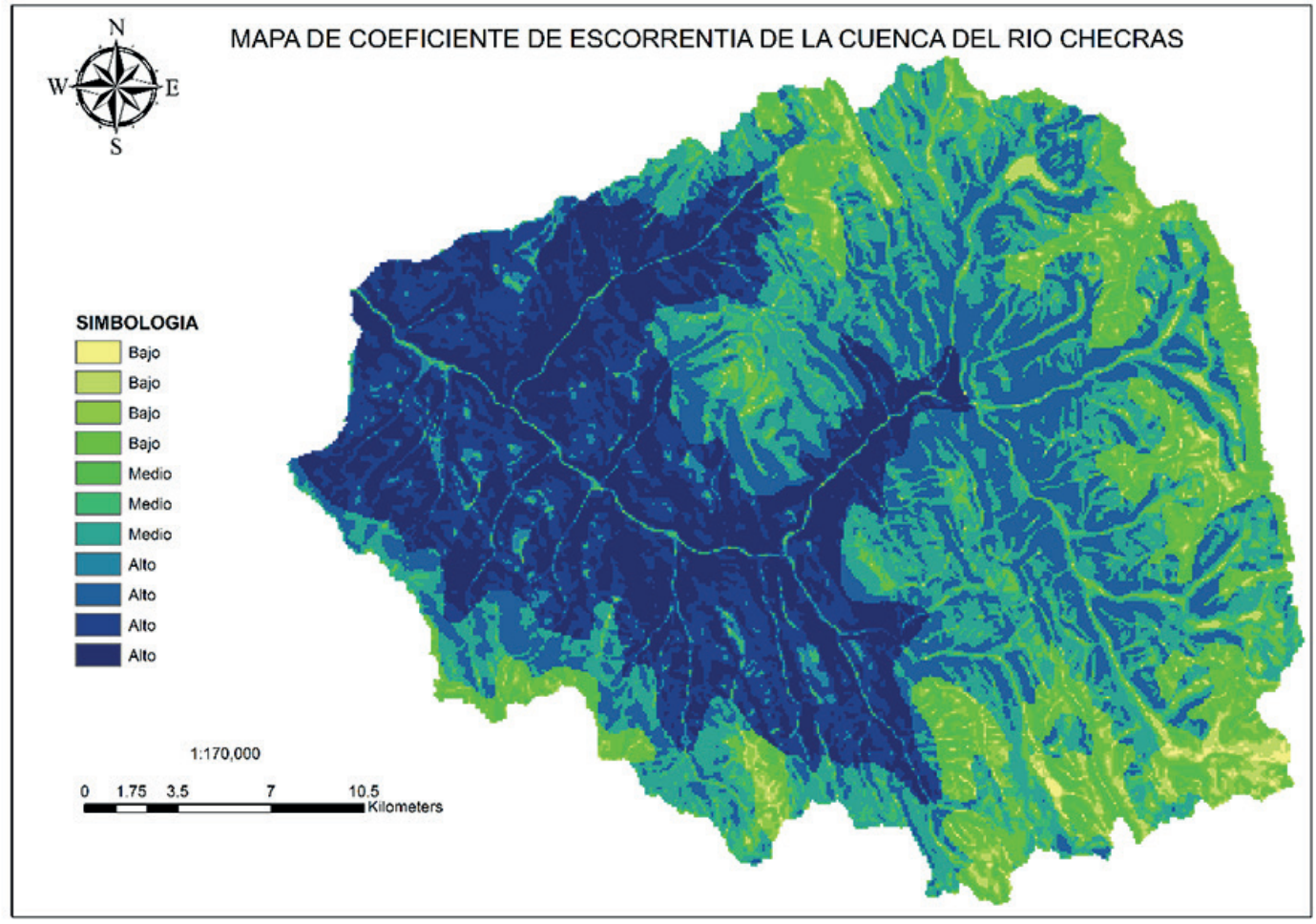

Figura 1. Mapa de coeficiente de escorrentía. Fuente: Elaboración propia.

Tabla 3. Precipitación media según el Método Aritmético

\begin{tabular}{clccc}
\hline Id & Estación & Altitud & $\begin{array}{c}\text { Método aritmético } \\
\text { Altura precipitación }\end{array}$ & Precipitación media \\
\hline 1 & Picoy & 2903 & 627.46 & \\
2 & Parquin & 3571 & 790.45 & \\
3 & Puñun & 2925 & 512.5 & 713.435 \\
4 & Sharin & 3660 & 754.1 & \\
5 & Yuracyacu Alto & 4120 & 783.4 & \\
6 & Cochaquillo & 4575 & 812.7 & \\
& & Suma & 4280.61 & \\
\hline
\end{tabular}

Elaboración propia. Fuente SENAMHI 
Tabla 4. Precipitación media según el Método Isoyetas

\begin{tabular}{cccccc}
\hline & \multicolumn{3}{c}{ Método de las Isoyetas } \\
Isoyeta & Isoyeta media & Área & \multicolumn{2}{c}{ \% área } & Altura de precipitación (mm) \\
\hline $1000-1050$ & 1025 & 2.37 & 0.3 & 0.003 & 2.96 \\
$950-1000$ & 975 & 15.93 & 1.9 & 0.019 & 18.94 \\
$900-950$ & 925 & 43.74 & 5.3 & 0.053 & 49.31 \\
$850-900$ & 875 & 80.87 & 9.9 & 0.099 & 86.24 \\
$800-850$ & 825 & 147.48 & 18.0 & 0.180 & 148.29 \\
$750-800$ & 775 & 108.35 & 13.2 & 0.132 & 102.35 \\
$700-750$ & 725 & 62.03 & 7.6 & 0.076 & 54.81 \\
$650-700$ & 675 & 63.59 & 7.8 & 0.078 & 52.32 \\
$600-650$ & 625 & 63.61 & 7.8 & 0.078 & 48.45 \\
$550-600$ & 575 & 76.07 & 9.3 & 0.093 & 53.31 \\
$500-550$ & 525 & 106.15 & 12.9 & 0.129 & 67.92 \\
$450-500$ & 475 & 50.28 & 6.1 & 0.061 & 29.11 \\
& Área total & 820.48 & & Precip.media & 714.01 \\
\hline
\end{tabular}

Elaboración propia. Fuente SENAMHI

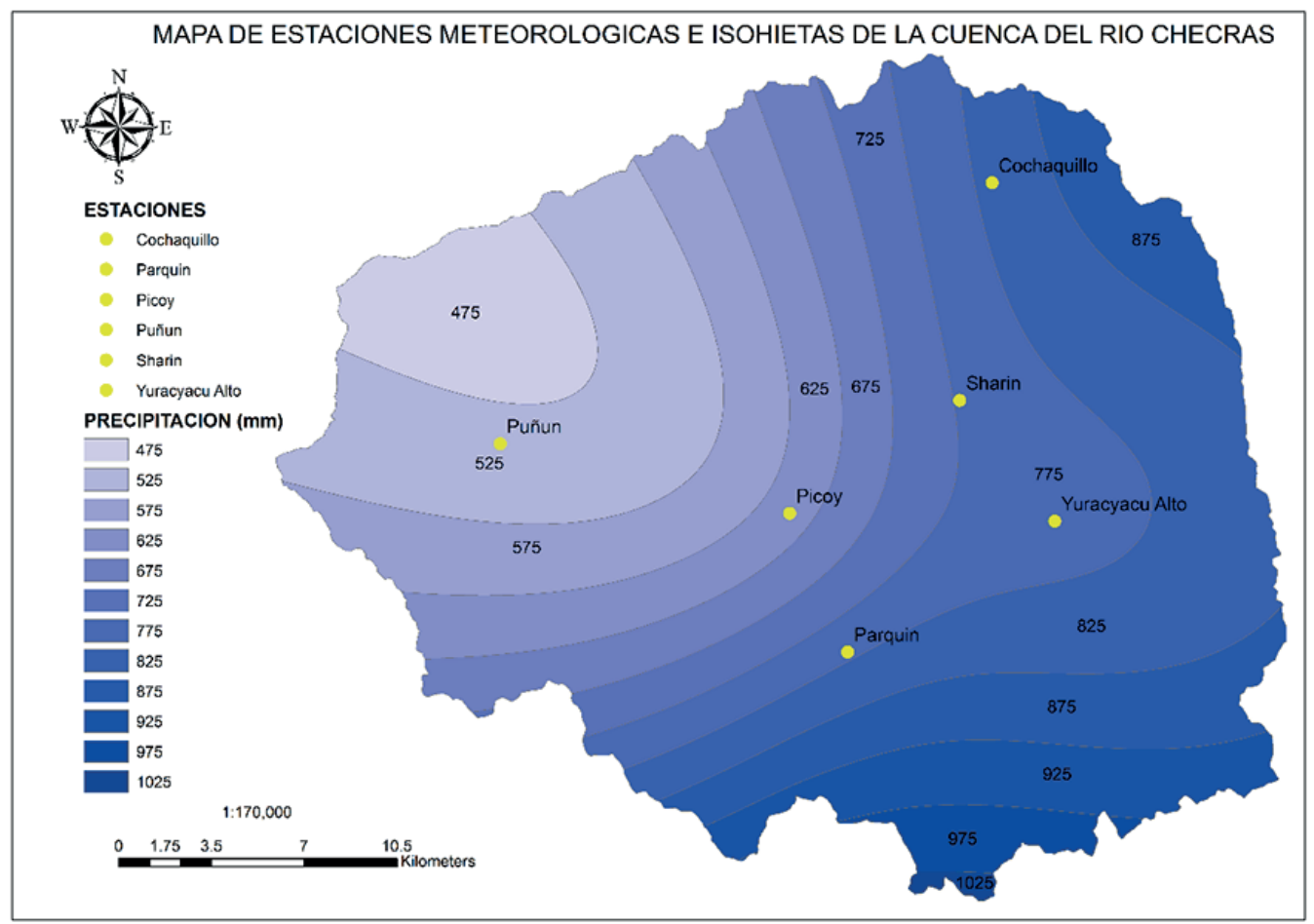

Figura 2. Mapa de isoyetas d la cuenca del río Checras

Después de calcular la precipitación media máxima, se determina la serie de precipitaciones de diseño y las curvas de Intensidad- Duración- Frecuencia (IDF), para diferentes periodos de retorno (Tabla 6 y Figura 3). Una vez conocida las series de intensidades de precipitación de diseño, se obtienen las curvas IDF para diferentes tiempos de retorno, el mismo que servirá para hallar los caudales máximos con el Método Racional.

\subsection{Estimación de caudales medios y caudales máximos}

Sabiendo que la cuenca del rio Checras tiene una extensión de $820.55 \mathrm{~km}^{2}$, con una precipitación media de $714 \mathrm{~mm}$ y con coeficiente de escorrentía ponderado igual a 0.54 , se puede calcular los caudales máximos, mínimos y medios de la cuenca, con ecuaciones que enlazan estos parámetros, como la ecuación sintética y la ecuación racional. 
Tabla 5. Precipitaciones máximas de 24 horas

\begin{tabular}{ccccc}
\hline $\mathbf{0}$ & Año & PP Max.24 horas & PPmax-PP & (PPmax-PP)Exp2 \\
\hline 1 & 1966 & 20.0 & -3.4 & 11.56 \\
2 & 1967 & 28.0 & 4.6 & 21.16 \\
3 & 1968 & 32.4 & 9.0 & 81 \\
4 & 1969 & 18.2 & -5.2 & 27.04 \\
5 & 1970 & 13.7 & -9.7 & 94.09 \\
6 & 1971 & 15.0 & -8.4 & 70.56 \\
7 & 1972 & 39.4 & 16.0 & 256 \\
8 & 1973 & 23.4 & 0.0 & 0 \\
9 & 1974 & 18.9 & -4.5 & 20.25 \\
10 & 1975 & 20.0 & -3.4 & 11.56 \\
$\ldots$ & $\ldots$ & $\ldots$ & $\ldots$ & $\ldots$ \\
120 & 2000 & 20.3 & -3.1 & 9.61 \\
121 & 2001 & 19.5 & -3.9 & 15.21 \\
122 & 2002 & 22.1 & -1.3 & 1.69 \\
123 & 2003 & 26.6 & 3.2 & 10.24 \\
124 & 2004 & 16.6 & -6.8 & 46.24 \\
125 & 2005 & 15.9 & -7.5 & 56.25 \\
126 & 2006 & 20.6 & -2.8 & 7.84 \\
127 & 2007 & 22.2 & -1.2 & 1.44 \\
128 & 2008 & 20.7 & -2.7 & 7.29 \\
\hline
\end{tabular}

Fuente: SENAMHI

Tabla 6. Series de intensidades de precipitación de diseño

\begin{tabular}{ccccccccc}
\hline Tr & \multicolumn{3}{c}{ Precipitaciones $\mathbf{( m m}$ ) } & \multicolumn{3}{c}{ Intensidades diseño $(\mathbf{m m} / \mathbf{h r})$} \\
\hline & 15 & 30 & 60 & $\mathrm{Tc}=89.645$ & 15 & 30 & 60 & $\mathrm{Tc}=90$ \\
25 & 13.17 & 15.662 & 18.625 & 20.591 & 52.68 & 26.34 & 13.17 & 13.782 \\
50 & 14.437 & 17.168 & 20.417 & 22.573 & 57.748 & 28.874 & 14.437 & 15.108 \\
250 & 17.351 & 20.633 & 24.537 & 27.128 & 69.404 & 34.702 & 17.351 & 18.157 \\
500 & 18.601 & 22.12 & 26.306 & 29.083 & 74.404 & 37.202 & 18.601 & 19.465 \\
\hline
\end{tabular}

Fuente: Elaboración propia.

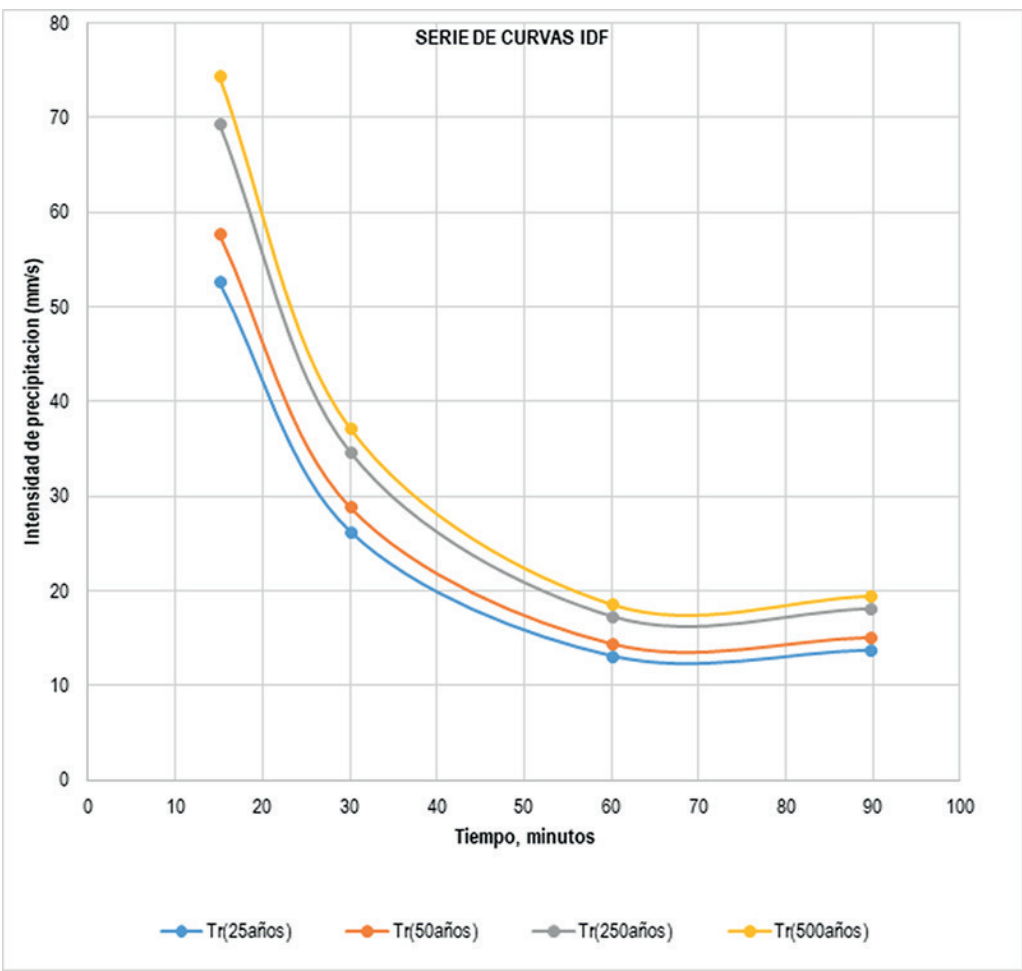

Figura 3. Serie de curvas IDF, según periodo de retorno de los eventos máximos.

Fuente: Elaboración propia. 
Integrando las ecuaciones de Voscresiensky, K. (1956), Zhelezniakov, G (1984) y Zhivotovsky, B. (1978), en una ecuación sintética, el volumen promedio de precipitaciones anuales para cuenca del rio Checras, expresado en $\mathrm{m}^{3} / \mathrm{s}$, es igual a $18.578 \mathrm{~m}^{3} / \mathrm{s}$ que se simplifica a partir del valor del volumen promedio de escurrimiento:

$$
\begin{gathered}
V=1000 * \bar{P} A \\
\mathrm{~V}=1000 * 714 * 820.55 \\
\mathrm{~V}=585872700
\end{gathered}
$$

Dónde:

V: Volumen promedio de escurrimiento

A: Área de la cuenca en $\mathrm{km}^{2}$

$\bar{P}$ : Precipitación promedio de la cuenca en $\mathrm{mm}$

El volumen de escurrimiento de la cuenca del rio Checras, convertido a caudal volumétrico, es de $18.578 \mathrm{~m}^{3} / \mathrm{s}$. De esta cantidad hay un porcentaje que forma el caudal promedio de la cuenca en su colector principal y la otra cantidad se diluye por evaporación y/o infiltración. Esto se comprueba con la siguiente ecuación:

$$
\begin{aligned}
& Q m=\frac{31.71 * C \bar{P} A}{10^{6}} \\
& Q m=\frac{31.71 * 0.5 * 714 * 820.55}{10^{6}}=10.032 \mathrm{~m}^{3} / \mathrm{s}
\end{aligned}
$$

\section{Dónde:}

Qm: Caudal medio de la cuenca $\mathrm{m}^{3} / \mathrm{s}$

A: $\quad$ Área de la cuenca en $\mathrm{km}^{2}$

$\bar{P}$ : $\quad$ Precipitación promedio de la cuenca en $\mathrm{mm}$

La otra ecuación simplificada que permite calcular el caudal medio de la cuenca es hacerlo únicamente en función del área, a través de la expresión:

$$
Q m=a A^{B}
$$

Donde a y b son variables, cuyos valores están dados en la siguiente relación:

$$
a=\frac{31.71 * C \bar{P}}{10^{6}} ; b=1
$$

Entonces el valor de $a$ es:

$$
a=\frac{31.71 * 0.540 * 714}{10^{6}}=0.0122
$$

Por lo tanto el caudal medio de la cuenca del rio Checras es de:

$$
Q m=0.0122 * 820.55^{1}=10.010 \mathrm{~m} 3 / \mathrm{s}
$$

En lo que respecta el caudal mínimo para la cuenca del rio Checras, se puede determinar mediante la siguiente ecuación:

$Q \min =\frac{\bar{P} A}{10^{6}}=$ Qeco $\quad Q \min =\frac{714 * 820.55}{10^{6}}=0.585 \mathrm{~m}^{3} / \mathrm{s}$

Este valor también representa el caudal ecológico (según la Autoridad Nacional del Agua), el cual alcanza los 585 litros de agua fluvial.

Por otro lado, para determinar los caudales máximos de cuencas extensas como Checras, se utiliza la ecuación racional modificada, que establece la siguiente relación:
$\mathrm{Qd}=0.278 \mathrm{CIAK}$
Dónde:
C: Coeficiente de escorrentía ponderado
I: Intensidad máxima de precipitación
$\mathrm{K}$ : Coeficiente de uniformidad
A: Área de la cuenca

Para el Método Racional Modificado de Témez, $\mathrm{K}$ es el coeficiente de uniformidad que busca corregir el tiempo de concentración de la cuenca para tener en cuenta la variación de la precipitación neta a lo largo del tiempo, según la siguiente ecuación:

$$
K=1+\frac{T c^{1.25}}{T c^{1.25}+14}
$$

Dónde:

$\mathrm{K}$ : Coeficiente de uniformidad

Tc: Tiempo de concentración

El tiempo de concentración calculado para la cuenca del rio Checras, con la fórmula de Kirpish es de 170 minutos aproximadamente.

$$
\begin{aligned}
& T_{c}=0.0195 * \frac{L^{0.77}}{S^{0.385}} \\
& T_{c}=0.0195 * \frac{39546^{0.77}}{0.09^{0.385}}=170.7 \mathrm{~min}
\end{aligned}
$$

Dónde:

Tc: Tiempo de concentración (min)

L: Longitud del cauce principal

S: Pendiente media del cauce principal

Luego, conociendo el tiempo de concentración de la cuenca, el coeficiente de uniformidad tiene el siguiente valor:

$$
K=1+\frac{89.645^{1.25}}{89.624^{125}+14}=1.9
$$

Finalmente, conocido los siguientes datos: área de la cuenca $=820.553 \mathrm{~km}^{2}$, coeficiente de escorrentía $=0.54$, 
coeficiente de uniformidad $=1.95$ y con precipitaciones máximas de diferentes duraciones (Tabla 10), se tiene las siguientes series de caudales máximos (Tabla 7, Tabla 8) así como las curvas de duración de caudales y la curva masa (Figura 5, Figura 6)

$$
Q d=0.278 C I A K
$$

$$
Q_{d}=\frac{0.278 * 0.54 * 52.68 * 820.55 * 1.95}{3600} * 1000
$$

\section{RESULTADOS}

En la presente investigación se comprueba la efectividad de la ecuación sintética y de la ecuación racional para determinar los caudales medios y caudales máximos respectivamente. Esta aplicación metodológica fue validada por Sandoval y Aguilera (2014), en sus estudios en las cuencas del Ecuador que carecen de información hidrológica. Por lo que este método es también idóneo para cálculos de caudales en cuencas altoandinas del Perú de hasta $1000 \mathrm{~km}^{2}$ que no tienen o tienen pocas estaciones meteorológicas e hidrológicas, como es el caso de la cuenca del río Checras.

La efectividad de la ecuación sintética de Voscresiensky, K. (1956), Zhelezniakov, G (1984) y Zhivotovsky, B. (1978) para la estimación de la precipitación media y del caudal medio de la cuenca del rio Checras alcanza el $94.3 \%$ por lo que es un método válido para cuencas de escasa información hidrológica y climática, que toma como variable principal el área de la cuenca. En efecto el caudal medio del rio Checras obtenido con las dos ecuaciones sintéticas son, respectivamente 10.032 y $10.010 \mathrm{~m}^{3} / \mathrm{s}$. El caudal promedio calculado por métodos experimentales de aforo y medición directa por SN Power y el SENAMHI, para la cuenca del rio Checras, a lo largo de 42 años, entre 1966 y 2008 , es de $9.4 \mathrm{~m}^{3} / \mathrm{s}$, por

Tabla 7. Caudales máximos- Método Racional Modificado de Témez

\begin{tabular}{lcccc}
\hline Qdiseño & $\mathbf{1 5}$ & $\mathbf{3 0}$ & $\mathbf{6 0}$ & $\mathbf{9 0}$ \\
\hline Qd(25 años $)$ & 35.43 & 17.72 & 8.86 & 9.27 \\
Qd(50 años $)$ & 38.84 & 19.42 & 9.71 & 10.16 \\
Qd(250 años $)$ & 46.68 & 23.34 & 11.7 & 12.21 \\
Qd(500 años $)$ & 50.04 & 25.02 & 12.51 & 13.09 \\
\hline
\end{tabular}

Fuente: Elaboración propia.

Tabla 8. Caudales máximos- Método Racional Clasico

\begin{tabular}{lcccc}
\hline Qdiseño & $\mathbf{1 5}$ & $\mathbf{3 0}$ & $\mathbf{6 0}$ & $\mathbf{9 0}$ \\
\hline Qd(25 años) & 64.839861 & 32.419931 & 16.20996525 & 16.96323 \\
Qd(50 años) & 71.0776821 & 35.538841 & 17.76942053 & 18.5953041 \\
Qd(250 años) & 85.4241783 & 42.712089 & 21.35604458 & 22.3480895 \\
Qd(500 años) & 91.5783033 & 45.789152 & 22.89457583 & 23.9580086 \\
\hline
\end{tabular}

Fuente: Elaboración propia.

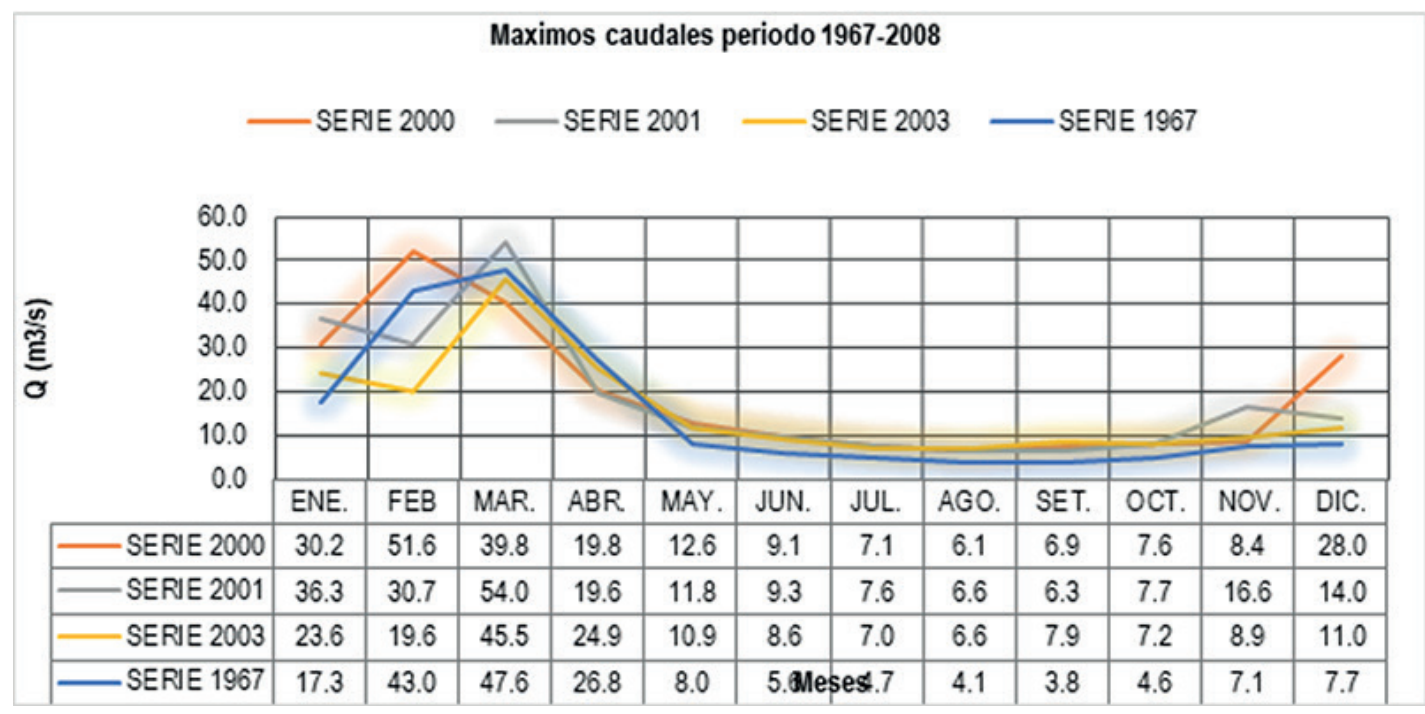

Figura 4. Serie de caudales máximos del rio Checras ocurridos entre 1967 y 2008 


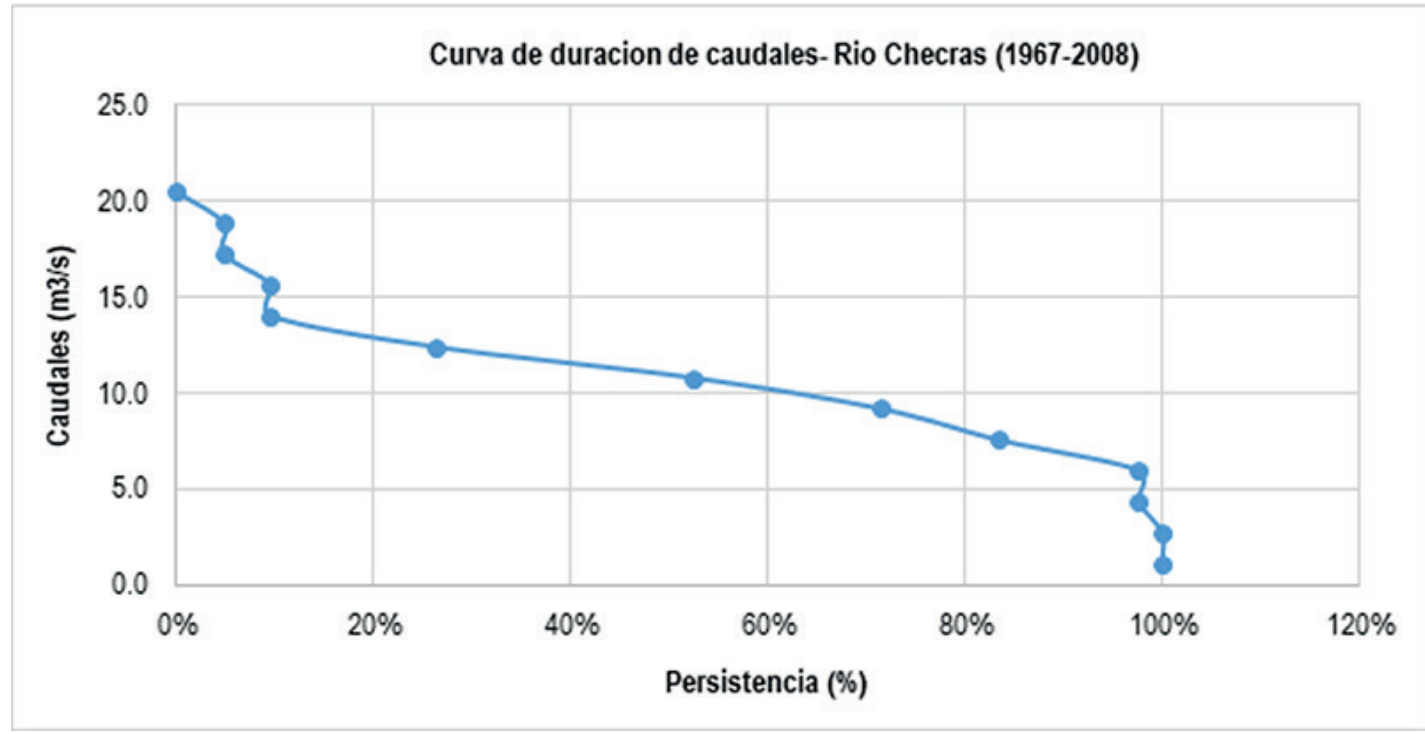

Figura 5. Curva de duración de caudales del rio Checras.

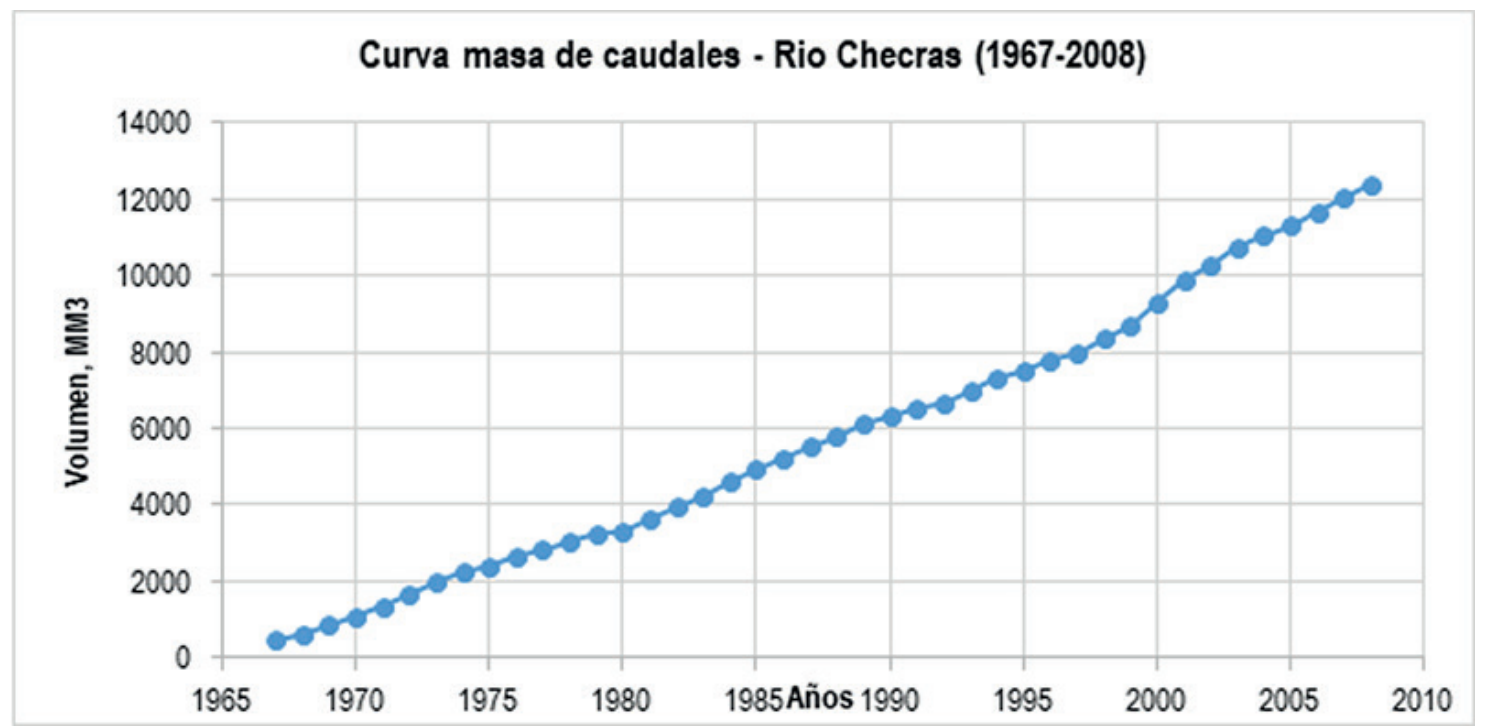

Figura 6. Curva masa del rio Checras.

lo que la diferencia excedente es de $0.6 \mathrm{~m}^{3} / \mathrm{s}$; es decir un margen de error de $5.99 \%$.

\section{DISCUSIÓN}

Integrando las ecuaciones, en una ecuación sintética, el volumen promedio de precipitaciones anuales para cuenca del rio Checras, expresado en $\mathrm{m}^{3} / \mathrm{s}$, es igual a $18.578 \mathrm{~m}^{3} / \mathrm{s}$ que se simplifica a partir del valor del volumen promedio de escurrimiento. En este caso el caudal medio de la cuenca representa el 53.8\% del caudal volumétrico del volumen de escurrimiento de la cuenca. Este es un valor que se ajusta a los cálculos del SENAMHI.

Las ecuaciones sintéticas también pueden determinar directamente el caudal mínimo de la cuenca, el mismo que representa su caudal ecológico. Para la cuenca del rio
Checras esto es de $0.585 \mathrm{~m}^{3} / \mathrm{s}$, la misma que fue corroborada mediante una medición directa, por SN Power, para la construcción de la Hidroeléctrica de Cheves (2014).

Con la información histórica de caudales medios, año por año entre 1967 y 2008, se construyó la curva masa de descarga de la cuenca del rio Checras y la curva de duración de caudales (CDC).

La curva masa, llamada también curva de volúmenes acumulados, es una curva que se utiliza en el estudio de regularización de los ríos por medio de embalses (Chereque, 1997).

El rio Checras descarga anualmente alrededor de 300 $\mathrm{MM}^{3}$ de agua, el cual es suficiente para abastecer de agua potable a más 500000 personas e irrigar hasta 50000 ha. Por eso la empresa SN Power ha construido una gigantesca 
represa para almacenar agua (del rio Checras y rio Churin) y abastecer a una de las hidroeléctricas más grandes de la cuenca del Pacifico, la hidroeléctrica de Cheves que produce $170 \mathrm{Mw}$ de energía.

La curva de duración de caudales es una curva de frecuencia acumulada, que presenta el porcentaje del tiempo que un determinado valor de caudal es igualado o excedido durante el periodo analizado y representa de forma simple y directa el rango de variación de caudales en un punto específico de una corriente (Searcy, 1963). La curva de duración de caudales (CDC) de la cuenca del rio Checras, indica una alta eficiencia hidráulica ya que durante 7 meses del año presenta caudales superiores a $10 \mathrm{~m}^{3} / \mathrm{s}$. Este caudal es un indicador muy importante para planificar proyectos agrícolas de miles de hectáreas, pues tendrían agua suficiente disponible durante gran parte del año.

Por otro lado, para la localización y dimensionamiento de obras hidráulicas y en general para todas las obras de ingeniería civil que interactúa con el agua, se requiere conocer el periodo de retorno de los grandes eventos, el mismo que está relacionado con los caudales máximos, llamado también caudales de diseño.

Los caudales máximos, son caudales extraordinarios de gran magnitud que se manifiestan en largos ciclos de periodos de retorno. La estimación de estos caudales es fundamental para dimensionar sistemas de drenaje, muros de encausamiento para proteger sistemas urbanos, alcantarillados, vertederos de demasías, luz en puentes, represas (Bateman, 2007)

El año 2010, la Autoridad Nacional del Agua (ANA), estimó las siguientes series de caudales, para periodos de retorno de 20, 50 y 200 años. Estos valores tienen una correlación más aproximada con la serie de caudales máximos obtenidos con el Método Racional Clásico que no considera el coeficiente de uniformidad. En este caso los caudales máximos para un periodo de retorno de 25 y 50 años son respectivamente, 64 y $71 \mathrm{~m}^{3} / \mathrm{s}$, para una duración de 15 minutos.

Estos caudales máximos para diferentes periodos de retorno se han calculado tomando en cuenta los siguientes parámetros de la cuenca: área de la cuenca igual a 820.55 $\mathrm{km}^{2}$, coeficiente de escorrentía 0.54, coeficiente de uniformidad 1.95 y precipitaciones máximas de diferentes duraciones.

\section{CONCLUSIONES}

1. Existe una integración de parámetros fundamentales de la cuenca en las ecuaciones sintéticas y ecuaciones racionales para la estimación de caudales máximos, caudales mínimos y caudales promedios de las cuencas.

2. Las ecuaciones sintéticas y ecuaciones racionales pueden ser aplicados con bastante eficiencia en la caracterización hidrológica de las cuencas con poca información hidrológica como es el caso de la cuenca del rio Checras.
3. La estimación de series de caudales máximos para la cuenca del rio Checras, es fundamental para definir la localización, extensión y dimensionamiento de obras de infraestructura física

4. El cálculo de los caudales medios anuales permite hallar el volumen de descarga de la cuenca graficada en curvas masa y por otro lado la curva de duración de caudales permite identificar, el caudal ecológico así como el caudal 70, 90, $100 \%$ de los 365 días del año, importantes para la planificación agrícola y urbana.

5. La gran extensión de la cuenca de Checras así como su máxima longitud determinan caudales de gran magnitud de hasta $54 \mathrm{~m}^{3} / \mathrm{s}$ dentro de un periodo de retorno de 50 años. La cuenca del rio Checras tiene gran eficiencia hidráulica. Supera a la cuenca del rio Chillón a pesar que esta tiene una extensión de $2444 \mathrm{~km}^{2}$, frente a $820.53 \mathrm{del}$ rio Checras. El caudal medio del rio Chillón es $4.2 \mathrm{~m}^{2} / \mathrm{s}$, según los registros de SENAMHI.

\section{AGRADECIMIENTOS}

Nuestro agradecimiento a la Universidad Nacional Mayor de San Marcos, nuestra alma mater donde aprendimos a desarrollar los principios de la investigación científica y que en esta ocasión nos permite desarrollar el presente artículo. También expresar nuestra gratitud a todos los colegas sanmarquinos por su mística y preocupación permanente por la ciencia y la investigación.

\section{REFERENCIAS BIBLIOGRÁFICAS}

Autoridad Nacional del Agua, (2010). Evaluación de recursos hídricos superficiales en la cuenca del río Huaura. Ministerio de Agricultura. Recuperado de https://hdl.handle. net/20.500.12543/00.12543/1965

Bateman, L. (2007). Hidrología Básica y Aplicada. Grupo de Investigación en Transporte de Sedimentos. GITS. Universidad Politécnica de Cataluña. Recuperado de https:// www.upct.es/ minaeees/hidrologia.pdf

Chereque, W (1985). Hidrología para estudiantes de ingeniería. Universidad Pontificia Católica del Perú- CONCYTEC. Recuperado de http://repositorio.pucp.edu.pe/index/ bitstream/handle/123456789/28689/hidrologia.pdf

Cheves (2014). Estudio de Impacto Ambiental de la cuenca del rio Churin-cuenca del rio Huaura. Proyecto Hidroenergético de Cheves. Recuperado de https://www.oefa.gob.pe/?wpfb $\mathrm{dl}=19794$

INGEMMET, (2008). Estudio Geoambiental de la Cuenca del rio Huaura. Instituto Geológico Minero y Metalúrgico. Recuperado de https://hdl.handle.net/20.500.12544/246

Sandoval Erazo Washington y Aguilera Ortiz Eduardo (2014). Determinación de Caudales en cuencas con poca información Hidrológica. Revista Ciencia UNEMI. https:// doi.org/10.29076/issn.2528-7737vol7iss 12.2014pp100$110 \mathrm{p}$ 
Searcy, J. (1963) Manual of Hydrology: Part 2, Low-Flow Techniques. Flow-Duration Curve. United States Department of the Interior. Geological Survey. Washington. Recuperado de https://pubs.usgs.gov/wsp/1542a/report.pdf

Searcy, J. (1969) Curva de dobles masas: manual de hidrología parte I: técnica de hidrología superficial. SIAC Sistema de Información Ambiental de Colombia

Robredo, J. et al. (2012). Un método para la estimación de caudales continuos en pequeñas cuencas de montaña. Ecología, No 24, 2012, pp. 27-42. Recuperado de https://www.miteco.gob.es/es/parques-nacionales-oapn/ publicaciones/ecologia_24_02_tcm30-100368.pdf
Villon, Maximo. (2011). Hidrología. Instituto Tecnológico de Costa Rica. República de Costa Rica.

Voscresiensky, K. (1956). Cálculos hidrológicos para el diseño de obras hidráulicas en pequeños ríos y riachuelos. Leningrado (San Petersburgo), Rusia. Ed. Hidrometereológica

Zhelezniakov, G. Negovskaya, T. y Ovcharov, E. (1984). Hidrología y regulación de escorrentía. Moscú, Rusia. Editorial Kolos.

Zhivotovsky, B. (1978). Cálculos hidrológicos para construcciones hidráulicas. Moscú, Rusia. Ed. UAP. 